\section{LENVATINIB AND PEMBROLIZUMAB IN ADVANCED ENDOMETRIAL CARCINOMA (EC): LONG-TERM EFFICACY AND SAFETY UPDATE FROM A PHASE 1B/2 STUDY}

${ }^{1}$ Vicky Makker*, ${ }^{2}$ Carol Aghajanian, ${ }^{3}$ Allen Cohn, ${ }^{4}$ Margarita Romeo, ${ }^{5}$ Raquel Bratos, ${ }^{6}$ Marcia Brose, ${ }^{7}$ Mark Messing, ${ }^{8}$ Lea Dutta, ${ }^{8}$ Corina Dutcus, ${ }^{8}$ Jie Huang, ${ }^{9}$ Emmett Schmidt, ${ }^{9}$ Robert Orlowski, ${ }^{10}$ Matthew Taylor. ${ }^{1}$ Memorial Sloan Kettering Cancer Center, Weill Cornell Medical Center, New York, NY, USA; ${ }^{2}$ Memorial Sloan Kettering Cancer Center, New York, NY, USA; ${ }^{3}$ Rocky Mountain Cancer Center, Denver, CO, USA; ${ }^{4}$ Catalan Institute of Oncology, Badalona, Spain; ${ }^{5} \mathrm{MD}$ Anderson Cancer Center España, Madrid, Spain; ${ }^{6}$ Abramson Cancer Center, University of Pennsylvania, Philadelphia, PA, USA; ${ }^{7}$ Texas Oncology, Bedford, TX, USA; ${ }^{8}$ Eisai Inc., Woodcliff Lake, NJ, USA; ${ }^{9}$ Merck and Co. Inc., Kenilworth, NJ, USA; ${ }^{10}$ Earle A. Chiles Research Institute, Providence Cancer Institute, Portland, OR, USA

Background Lenvatinib is a multikinase inhibitor of VEGFR 1-3, FGFR 1-4, PDGFR $\alpha$, RET, and KIT. Pembrolizumab is an anti-programmed death-1 monoclonal antibody. We previously reported results from a cohort of 108 patients with metastatic EC (data cutoff date, January 10, 2019) who received lenvatinib + pembrolizumab as part of an ongoing multicenter, open-label, phase $1 \mathrm{~b} / 2$ study evaluating the combination treatment in patients with selected solid tumors (NCT02501096). Lenvatinib + pembrolizumab showed a tolerable safety profile and promising antitumor activity per immune-related (ir) Response Evaluation Criteria In Solid Tumors (RECIST) by investigator assessment, including an objective response rate (ORR) of 38.9\% (95\% confidence interval [CI], 29.7-48.7), median progression-free survival (PFS) of 7.4 months (95\% CI, 5.3-8.7), and median overall survival (OS) of 16.7 months (95\% CI, 15.0-not estimable). ${ }^{1}$ Here we present updated efficacy and safety data (data cutoff date: August 18, 2020).

Methods Patients included in the EC cohort had histologically confirmed, measurable metastatic EC and had received $\leq 2$ prior chemotherapies (unless discussed with the sponsor). Patients received lenvatinib ( $20 \mathrm{mg}$ orally once daily) and pembrolizumab (200 mg intravenously once every 3 weeks). The phase 2 efficacy endpoints included ORR, PFS, OS, and duration of response. Tumor assessments for primary and secondary endpoints were evaluated by investigators per irRECIST.

Results The 108 patients from the key efficacy analysis set for the previously reported results were all included in these updated analyses. Median follow-up duration for the study was 34.7 months. Efficacy outcomes are summarized in table 1. Treatment-related adverse events (TRAEs) occurred in 104 (96\%) patients (94 [87\%] grade $\leq 3,10$ [9\%] grade $\geq 4$ ). TRAEs led to study-drug interruption of 1 or both drugs in $80(74.1 \%)$ patients and dose reductions of lenvatinib in 73 $(67.6 \%)$ patients; $23(21.3 \%)$ patients discontinued 1 or both drugs due to a TRAE. The most common grade $\geq 3$ TRAEs were hypertension (33.3\%), lipase increased (9.3\%), fatigue $(8.3 \%)$, and diarrhea (7.4\%).

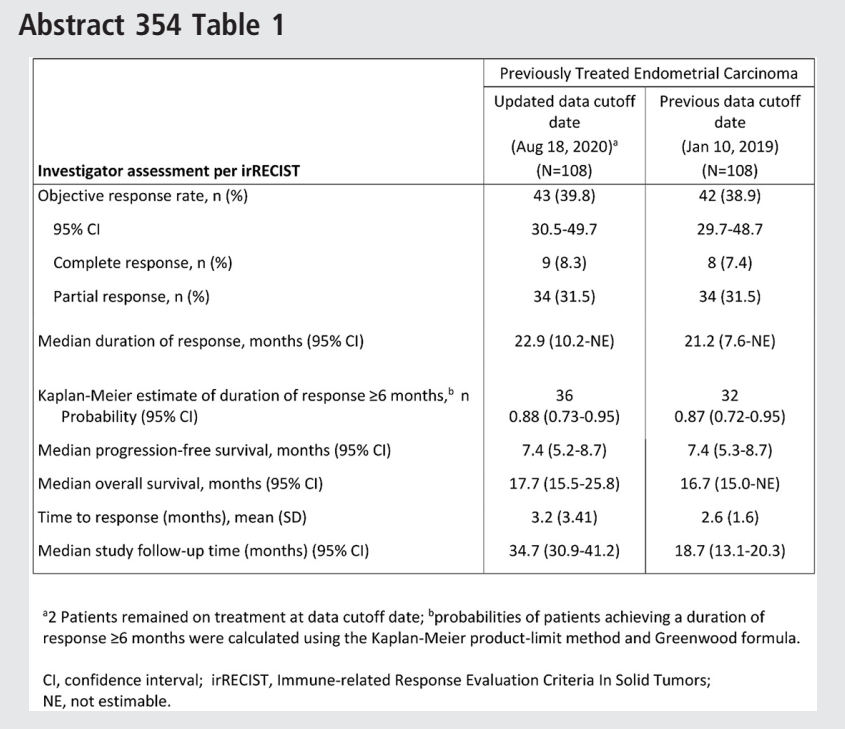

Conclusions With extended follow-up, our updated efficacy analysis continued to show clinical benefit in patients with metastatic EC who received lenvatinib + pembrolizumab. Moreover, the combination had a manageable safety profile that was generally consistent with the established safety profiles of the individual monotherapies. No new safety signals were detected. A phase 3 study of lenvatinib + pembrolizumab versus treatment of physician's choice in advanced endometrial cancer further supports the lasting clinical benefits observed in our study. ${ }^{2}$

Trial Registration www.clinicaltrials.gov NCT02501096

\section{REFERENCES}

1.. Makker V, Taylor MH, Aghajanian C, et al. Lenvatinib plus pembrolizumab in patients with advanced endometrial cancer. J Clin Oncol 2020;38(26):29812992.

2. Makker V, Colombo N, Casado Herráez A, et al. A multicenter, open-label, randomized, phase 3 study to compare

Ethics Approval This study was approved by the following ethics committees/institutional review boards (IRBs): Oregon Health \& Sciences University IRB, IntegReview IRB, Memorial Sloan Kettering Cancer Center IRB, University of Pennsylvania Office of Regulatory Affairs IRB, Dana-Farber Cancer Institute IRB, The University of Chicago Biological Sciences Division IRB, University of Texas MD Anderson Cancer Center IRB, Western IRB, Quorum Review IRB, US Oncology, Inc. IRB, CEIm - Comité de Ética de la Investigación con Medicamentos, Regional Komite for Medisinsk og Helsefagli Forskningsetikk, and REC - Regional Committees for Medical and Health Research Ethics. All participants gave informed consent before taking part in this study.

Consent No identifying information is contained in this abstract so no permission from participants is considered necessary.

http://dx.doi.org/10.1136/jitc-2021-SITC2021.354 